\title{
Modal finite element synthesis of acoustic boundary receptances
}

\author{
SAmir ASSAF ${ }^{1, a}$ And JeAn-Marie Lagache ${ }^{2}$ \\ 1 Laboratoire d'Acoustique et Vibration, École Supérieure des Techniques Aéronautiques et de Construction Automobile, \\ 34 rue Victor Hugo, 92300 Levallois-Perret, France \\ 2 Architecture Mécanique Moteurs et Transmissions, PSA, Centre de la Garenne, 18 rue des Fauvelles, \\ 92250 La Garenne Colombes, France
}

Received 30 September 2008, Accepted 18 December 2008

\begin{abstract}
The main issue in the present paper is to comment and illustrate on new acoustic examples the method of analysis of modal series, termed "method of orthocomplement", that has been recently proposed by the authors to improve the convergence of such series. The general method consists in a direct analysis and transformation of the remainders of ordinary series. It results in a family of "hybrid" modal representations involving an ordinary modal sum of order $N$, a "quasi-static" term based on the $N$ first modes, and an "accelerated" modal series. Using the transformed modal formulae eliminates the Gibbs oscillations - that are attached in infinite dimensional models to modal boundary discontinuities - and also the consequences of such phenomena on finite element approximations. The method is applied in the present paper to plane waves in acoustic tubes and to 3D acoustic fields inside a car compartment, in view of the synthesis of acoustic receptances or impedances to be used in practical acoustic design. The main technical difficulty being the treatment of singular linear boundary problems or systems of linear equations that arise during the study of closed rigid cavities or tubes, a whole section of the paper had thus to be devoted to pseudo-inversion.
\end{abstract}

Key words: Modal truncation / static correction / pseudo-inversion / Gibbs phenomenon / acoustics / finite-element

\begin{abstract}
Résumé - Synthèse modale par éléments-finis d'impédances acoustiques de parois. Le présent article se propose de commenter et d'illustrer sur de nouveaux exemples acoustiques la méthode d'analyse des séries modales, baptisée "méthode de l'orthocomplément ", qui a été récemment proposée par les auteurs pour améliorer la convergence de telles séries. La méthode générale repose sur une analyse directe des restes modaux. Il s'en déduit une famille de représentations modales « hybrides » incluant une somme modale ordinaire d'ordre $N$, un terme « quasi-statique $»$ construit à partir des $N$ premiers modes et enfin, une série modale « accélérée ». L'utilisation des représentations modifiées élimine les oscillations de Gibbs - attachées, dans les modèles analytiques, aux discontinuités-frontières des représentations modales - ainsi que les conséquences de ces phénomènes sur les approximations par éléments-finis. La méthode est ici appliquée à l'étude classique des ondes planes dans les tubes, puis à l'étude du champ acoustique tridimensionnel dans un habitacle automobile, avec pour objectif l'obtention des impédances ou réceptances nécessaires à la discussion des problèmes pratiques de design acoustique. La principale difficulté technique est liée aux singularités présentées, dans l'étude des cavités ou tubes fermés, par les problèmes aux limites et systèmes linéaires associés. Une section entière a donc dû être consacrée aux pseudo-inverses.
\end{abstract}

Mots clés : Troncature modale / correction statique / pseudo-inversion / phénomène de Gibbs / acoustique / éléments-finis

\section{Introduction}

A major difficulty in the analysis of acoustic cavities lies in that acoustic modes with zero modal boundary

\footnotetext{
${ }^{a}$ Corresponding author: sassaf@estaca.fr
}

velocities can only reconstitute non-zero boundary velocities in a weak $L^{2}$ sense. Indeed, carefully prepared infinite dimensional analytical examples present typical Gibbs phenomena [1] that are the mark of considerable errors of truncation, bad evaluations of impedances and inaccurate finite element approximations [2]. 
To control such phenomena, Ritz methods over extended "bases" have been since long proposed in the acoustic or structural fields [3-7]. A direct analysis of the modal remainder has however been performed in reference [8], leading to closed form modal representations including an ordinary modal sum of order $N$, a "pseudostatic" term based on the $N$ first modes, and an "accelerated" series with minored remainder and no boundary discontinuity. The proposed method - termed "method of orthocomplement" - is outlined in Section 2 of the present paper. Applying the method to the acoustic pressure, $p$, inside a region $\Omega$ subjected, at the circular frequency $\omega$, to an acceleration, $\gamma$, on the boundary $\partial \Omega$, results in the representation formula

$$
\begin{aligned}
p=\left\{\sum_{m=1}^{N} \psi_{m}\right. & \left.\frac{c^{2}}{\omega_{m}^{2}-\omega^{2}} \int_{\partial \Omega} \psi_{m} D \mathrm{~d} s\right\}+\eta_{N} \\
& +\left\{\sum_{m=N+1}^{\infty} \psi_{m} \frac{\omega^{2}}{\omega_{m}^{2}} \frac{c^{2}}{\omega_{m}^{2}-\omega^{2}} \int_{\partial \Omega} \psi_{m} D \mathrm{~d} s\right\}
\end{aligned}
$$

where $\rho$ denotes the fluid density; $c$ the sound speed; $\mathbf{n}$ the outer normal along $\partial \Omega ; D$ the normal quantity of acceleration

$$
D=-\rho \gamma \cdot \mathbf{n}
$$

$\psi_{m}, \omega_{m}, m=1, \ldots \infty$, the rigid cavity modes and associated eigenfrequencies; and $\eta_{N}$, a pseudo-static term, solution to the singular Neumann problem with incompressiblity condition

$$
\left\{\begin{aligned}
\nabla^{2} \eta_{N} & =\sum_{m=1}^{N} \psi_{m}\left(\int_{\partial \Omega} \psi_{m} D \mathrm{~d} s\right) \text { in } \Omega, \frac{\partial \eta_{N}}{\partial n}=D \\
\int_{\Omega} \eta_{N} \mathrm{~d} \nu & =0
\end{aligned}\right.
$$

Since a complete proof can be found in [8] it was here preferred, in view of practical applications, to apply the methodology to $1 \mathrm{D}$ acoustic tubes. Correct modal expressions of impedances and transfers in closed or open tubes are thus given in Sections 3.1.1 and 3.2.1. An interesting result is the expression of the static term in a closed tube as

$$
\eta_{1}=\frac{\rho \gamma}{2 L}\left(x^{2}-\frac{L^{2}}{3}\right)
$$

The effective study of $3 \mathrm{D}$ cavities is considered in Section 4. Numerical methods having to face the Helmholtz $0 \mathrm{~Hz}$-singularity, useful pseudo-inversion results are briefly recalled in Section 4.1.1. In Section 4.2, a special attention is devoted to the finite-element expression of the solution to problem (3) that was obtained in [8], namely

$$
\eta_{N}=\left(1-\Pi_{Q}\right)(\lambda \Pi+\mathbf{H})^{-1}\left(\mathbf{F}-\mathbf{G}_{N}\right)
$$

with $\lambda \neq 0$ an arbitrarily non-zero number; $\mathbf{H}$ and $\mathbf{Q}$, the classical acoustic matrices such that

$$
\int_{\Omega} \nabla f \nabla g \mathrm{~d} x=\mathbf{f} \mathbf{H} \mathbf{g}, \int_{\Omega} f g \mathrm{~d} x=\mathbf{f} \mathbf{Q} \mathbf{g}
$$

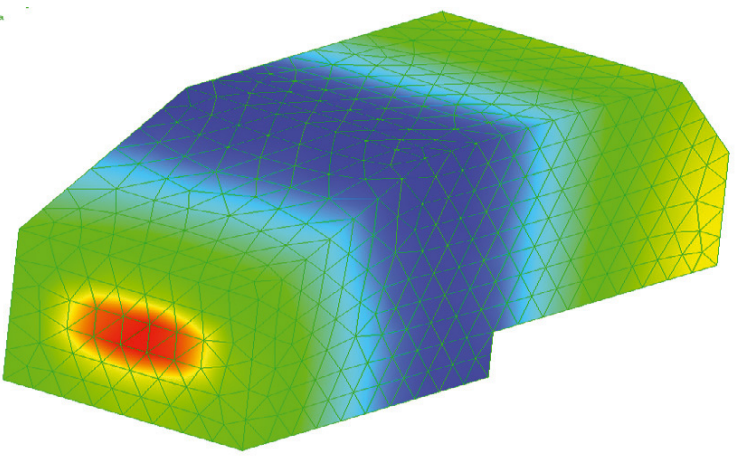

Fig. 1. Boundary source in a simplified car compartment.

$\boldsymbol{\Pi}$ and $\boldsymbol{\Pi}_{Q}$, the Euclidian and $\mathbf{Q}$-orthogonal projectors onto the nullspace, $\operatorname{Ker}(\mathbf{H})$; and $\mathbf{F}, \mathbf{G}_{N}$, "source vectors", respectively on $\partial \Omega$ and inside $\Omega$, such that

$$
\delta \eta^{\mathrm{T}} \mathbf{F} \simeq \int_{\partial \Omega} \delta \eta D \mathrm{~d} s, \delta \eta^{\mathrm{T}} \mathbf{G}_{N} \simeq \int_{\Omega} \delta \eta g_{N} \mathrm{~d} \nu
$$

A practical application of the preceding formulae to a $3 \mathrm{D}$ simplified car compartment is given in Section 4.3 (Fig. 1). As a conclusion, part 5 presents possible modal calculations of vibro-acoustic coupling of the car compartment with boundary resonators.

\section{Presentation of the method of orthocomplement}

The "method of orthocomplement" starts with the basic projection formula

$$
x=\sum_{m=1}^{N}\left\langle\Psi_{m} \mid x\right\rangle_{M} \Psi_{m}+\varepsilon_{N}=\sum_{m=1}^{N} \frac{\Psi_{m} \Psi_{m}^{T}}{\omega_{m}^{2}-\omega^{2}} \mathbf{f}+\varepsilon_{N},
$$

with $\Psi_{m}, m=1, \ldots N$, a M-orthonormal system of eigenmodes; $\langle. \mid .\rangle_{M}$, the scalar product induced by the mass operator $\mathbf{M}$ (or $\mathbf{Q}$-acoustic operator); $\varepsilon_{N}$, the orthocomplement $\left\langle\Psi_{m} \mid \varepsilon_{N}\right\rangle_{M}=0, m=1, \ldots N$.

The computation of this last term involves three steps :

(a) The first step - or forward step - consists in assembling the set of equations that characterises $\varepsilon_{N}$ and splitting it into two nested sub-problems, such that $\varepsilon_{N}=\eta_{N}+\rho_{N}$, with

$-\eta_{N}$, the solution to a pseudo-static problem relative to the static operator, $(\mathrm{A})$;

$-\rho_{N}$, the solution to a dynamical problem relative to the full dynamical operator, with the pseudo-static solution $\eta_{N}$ in the second member, (B). 
The consistency of the decomposition rests on the solvability of problem (A) which is thus the major technical task at the considered stage.

(b) In the second step - or coordination step -, modal relations offer a much more attractive alternative than solving (A) and assembling problem (B) to compute $\rho_{N}$.

Indeed, in case the static operator is not singular (Sect. 3.2.1), the modal projections of $\rho_{N}$ can be expressed in terms of the corresponding projections of $\eta_{N}:\left\langle\boldsymbol{\Psi}_{k} \mid \rho_{N}\right\rangle=f\left(\left\langle\boldsymbol{\Psi}_{k} \mid \eta_{N}\right\rangle\right.$. Surprisingly the projections $\left\langle\boldsymbol{\Psi}_{k} \mid \eta_{N}\right\rangle$ are directly computable, which makes that the modal projections of $\rho_{N}$ - and thus the complete solution to problem (B), - can be found by composition of the two preceding sets of formulae.

In case the static operator is singular (Sect. 3.1.1), and the solvability conditions are fulfilled, solution $\eta_{N}$ is not unique and both problems (A) and (B) suffer from indetermination. Modes at positive frequencies can bear the same treatment as before, but, due to unavoidable divisions by zero, the $\sigma$ modes at $0 \mathrm{~Hz}$ cannot. Complementary relations $\left\langle\boldsymbol{\Psi}_{k} \mid \eta_{N}\right\rangle=0, k=1, \ldots \sigma$, can then be added to problem (A) to render its solution $\eta_{N}$ unique, and annihilate the corresponding projections $\left\langle\boldsymbol{\Psi}_{k} \mid \rho_{N}\right\rangle=$ $f\left(\left\langle\Psi_{k} \mid \eta_{N}\right\rangle\right), k=1, \ldots \sigma$. A complete solution of problem (B) then becomes possible, since all other projections $\left\langle\boldsymbol{\Psi}_{k} \mid \rho_{N}\right\rangle=f\left(\left\langle\boldsymbol{\Psi}_{k} \mid \eta_{N}\right\rangle\right), k>\sigma$, are known.

(c) The third step - or backward step - finally consists in writing the solution as the superposition of an ordinary modal sum, the solution to the pseudostatic problem and the spectral development of $\rho_{N}$, which will be hereafter recognized as the "accelerated" modal series of formula (1).

\section{1D acoustics}

\subsection{Closed tube}

\subsubsection{General equations}

Plane waves inside an acoustic tube of length $L$, closed at $x=0$ and subjected to an acceleration $\gamma$ at $x=L$, are governed by the 1D Helmholtz equation and boundary conditions

$$
\frac{\mathrm{d}^{2} p}{\mathrm{~d} x^{2}}+k^{2} p(x)=0, \frac{\mathrm{d} p}{\mathrm{~d} x}(0)=0, \frac{\mathrm{d} p}{\mathrm{~d} x}(L)=-\rho \gamma
$$

where $\rho$ is the mass density; $c$, the sound speed; and $k=$ $\omega / c$, the wavenumber at the circular frequency $\omega$.

Normalized modes $\psi_{m}$ and eigenfrequencies $k_{m}$ satisfying the homogeneous equations

$$
\frac{\mathrm{d}^{2} \psi_{m}}{\mathrm{~d} x^{2}}+k_{m}^{2} \psi_{m}(x)=0, \frac{\mathrm{d} \psi_{m}}{\mathrm{~d} x}(0)=0, \frac{\mathrm{d} \psi_{m}}{\mathrm{~d} x}(L)=0
$$

and the normalization conditions $\left\langle\psi_{k} \mid \psi_{m}\right\rangle=\delta_{k m^{-}}$with $\langle f \mid g\rangle=\int_{[0, L]} f g \mathrm{~d} x$, the ordinary $L^{2}$-inner product, and $\delta_{k m}$, the Kronecker symbol - are readily identifiable to

$$
\begin{aligned}
\text { with } \psi_{m}(x) & = \begin{cases}\sqrt{\frac{1}{L}} & \text { if } \quad m=1 \\
\sqrt{\frac{2}{L}} & \text { otherwise }\end{cases}
\end{aligned}
$$

Green formulae - that in the considered 1D example merely result from adequate integrations by parts - can be written for any pair of regular functions

$$
\begin{aligned}
&\left\langle\frac{\mathrm{d}^{2} f}{\mathrm{~d} x^{2}} \mid g\right\rangle=\int_{[0, L]} \frac{\mathrm{d}^{2} f}{\mathrm{~d} x^{2}} g \mathrm{~d} x \\
&=\int_{[0, L]} \frac{\mathrm{d}}{\mathrm{d} x}\left(\frac{\mathrm{d} f}{\mathrm{~d} x} g\right) \mathrm{d} x-\int_{[0, L]} \frac{\mathrm{d} f}{\mathrm{~d} x} \frac{\mathrm{d} g}{\mathrm{~d} x} \mathrm{~d} x \\
&=\left[\frac{\mathrm{d} f}{\mathrm{~d} x} g\right]_{0}^{L}-\int_{[0, L]} \frac{\mathrm{d} f}{\mathrm{~d} x} \frac{\mathrm{d} g}{\mathrm{~d} x} \mathrm{~d} x=\cdots \\
& \ldots=\left[\frac{\mathrm{d} f}{\mathrm{~d} x} g\right]_{0}^{L}-\left\langle\frac{\mathrm{d} f}{\mathrm{~d} x} \mid \frac{\mathrm{d} g}{\mathrm{~d} x}\right\rangle \\
& \text { and }\left\langle\frac{\mathrm{d}^{2} f}{\mathrm{~d} x^{2}} \mid g\right\rangle-\left\langle\frac{\mathrm{d}^{2} g}{\mathrm{~d} x^{2}} \mid f\right\rangle=\left[\frac{\mathrm{d} f}{\mathrm{~d} x} g-\frac{\mathrm{d} g}{\mathrm{~d} x} f\right]_{0}^{L}
\end{aligned}
$$

\subsubsection{Analysis by the method of orthocomplement}

Let $p$ denote the solution to problem (9) and $N$ be an arbitrary positive integer. Substituting for the boundary values and derivatives of $p, \psi_{m}$, from conditions (9) and (10) into the second Green formula immediately brings $\left\langle p \mid \psi_{m}\right\rangle=\frac{-\rho \gamma \psi_{m}(L)}{\left(k_{m}^{2}-k^{2}\right)}$. The solution $p$, to Equation (9) can thus be expanded at any order $N$ as

$$
p(x)=\sum_{m=1}^{N}-\rho \gamma \frac{\psi_{m}(x) \psi_{m}(L)}{\left(k_{m}^{2}-k^{2}\right)}+\varepsilon_{N}
$$

with a remainder - or orthocomplement $-\varepsilon_{N}$, orthogonal to the first $N$ modes.

\section{a) Forward step}

The first and second derivatives of $\varepsilon_{N}$ with respect to the space coordinate, $x$, can be deduced from Equation (13). Using Equations (9), (10), then shows that the orthocomplement satisfies

$$
\begin{aligned}
\frac{\mathrm{d}^{2} \varepsilon_{N}(x)}{\mathrm{d} x^{2}}+k^{2} \varepsilon_{N}(x) & =-\sum_{m=1}^{N} \rho \gamma \psi_{m}(L) \psi_{m}(x), \\
\frac{\mathrm{d} \varepsilon_{N}}{\mathrm{~d} x}(0) & =0, \frac{\mathrm{d} \varepsilon_{N}}{\mathrm{~d} x}(L)=-\rho \gamma
\end{aligned}
$$


Let this problem be split up into two nested subproblems (A) and (B)

$$
\begin{aligned}
\varepsilon_{N}= & \eta_{N}+\rho_{N} \\
& \left\{\begin{array}{l}
\frac{\mathrm{d}^{2} \eta_{N}}{\mathrm{~d} x^{2}}=\sum_{m=1}^{N}-\rho \gamma \psi_{m}(L) \psi_{m}(x) \frac{\mathrm{d} \eta_{N}}{\mathrm{~d} x}(0)=0, \\
\frac{\mathrm{d} \eta_{N}}{\mathrm{~d} x}(L)=-\rho \gamma(\mathrm{A}) \\
\frac{\mathrm{d}^{2} \rho_{N}}{\mathrm{~d} x^{2}}+k^{2} \rho_{N}=-k^{2} \eta_{N} \frac{\mathrm{d} \rho_{N}}{\mathrm{~d} x}(0)=0, \\
\frac{\mathrm{d} \rho_{N}}{\mathrm{~d} x}(L)=0(\mathrm{~B})
\end{array}\right.
\end{aligned}
$$

The solution to the Neumann boundary problem (A), if there is one, can only be determined within an arbitrary constant. It should be known [9] that for a solution to exist the forcing terms, $g_{N}=\sum_{m=1}^{N} \rho \gamma \psi_{m}(L) \psi_{m}(x)$ and $D$, have to satisfy the "integrability condition"

$$
-\int_{[0, L]} g_{N} \mathrm{~d} x+[D]_{0}^{L}=0
$$

Owing to the fact that the acoustic mode at $0 \mathrm{~Hz}$ is a constant, condition (16) can be brought to the more suitable form $\int_{0}^{L} \psi_{1} \sum_{m=1}^{N} \rho \gamma \psi_{m}(L) \psi_{m}(x)+\left[\psi_{1} D\right]_{0}^{L}=0$ and be readily verified, since

$$
\begin{aligned}
& -\int_{[0,1]} \psi_{1} \sum_{m=1}^{N} \psi_{m} \psi_{m}(\mathrm{~L}) \mathrm{d} \nu+\left[\psi_{1} D\right]_{0}^{L} \\
& =-\sum_{m=1}^{N}\left(\int_{[0,1]} \psi_{1} \psi_{m} \psi_{m}(L) D \mathrm{~d} x\right)+\left[\psi_{1} D\right]_{0}^{L} \\
& =-\sum_{m=1}^{N} \delta_{1 m}\left[\psi_{m} D\right]_{0}^{L}+\left[\psi_{1} D\right]_{0}^{L}=0
\end{aligned}
$$

At the present stage of the discussion, problem (A) admits an infinite number of solutions, differing one from the other by an arbitrary constant.

\section{b) Coordination step}

Problem (B) is an ordinary dynamical problem with homogeneous boundary conditions. The modal expansion of $\rho_{N}$ can be written as

$$
\rho_{N}(x)=\sum_{m=1}^{\infty}\left\langle\rho_{N} \mid \psi_{m}\right\rangle \psi_{m}(x)
$$

Substituting for the boundary values and derivatives of $\rho_{N}, \psi_{m}$, from conditions (15) and (10) into the second Green formula for $\rho_{N}, \psi_{m}$, brings

$$
\left\langle\rho_{N} \mid \psi_{m}\right\rangle=\frac{k^{2}}{k_{m}^{2}-k^{2}}\left\langle\eta_{N} \mid \psi_{m}\right\rangle
$$

Similarly, substituting for the boundary values and derivatives of $\eta_{N}, \psi_{m}$, from the same conditions (15) and (10) into the second Green formula for $\eta_{N}, \psi_{m}$, brings

$$
k_{m}^{2}\left\langle\eta_{N} \mid \psi_{m}\right\rangle=-\rho \gamma\left[\psi_{m}(L)-\sum_{q=1}^{N} \delta_{m q} \psi_{q}(L)\right]
$$

which gives for any $m \neq 1$

$$
\left\langle\eta_{N} \mid \psi_{m}\right\rangle= \begin{cases}0 \quad \text { if } \quad 1<m \leq N \\ \frac{-\rho \gamma \psi_{m}(L)}{k_{m}^{2}} & m>N\end{cases}
$$

and the identity $0=0$ when $m=1$.

It now remains to be remarked that imposing the complementary condition

$$
\left\langle\eta_{N} \mid \psi_{1}\right\rangle=0
$$

fixes the unknown constant in the solution, $\eta_{N}$, to problem (A), and, simultaneously, fully determines the solution, $\rho_{N}$, to problem (B), since substituting Equations (19), (20) into Equation (18) yields

$$
\begin{gathered}
\left\langle\rho_{N} \mid \psi_{m}\right\rangle=\frac{k^{2}}{k_{m}^{2}-k^{2}}\left\langle\eta_{N} \mid \psi_{m}\right\rangle \\
=\left\{\begin{array}{l}
0 \quad \text { if } \quad 1 \leq m \leq N \\
-\frac{k^{2}}{k_{m}^{2}} \frac{\rho \gamma \psi_{m}(L)}{k_{m}^{2}-k^{2}} \quad \text { if } \quad m>N
\end{array}\right.
\end{gathered}
$$

Because they only differ from ordinary ones by factors $k^{2} / k_{m}^{2}$ tending to 0 with increasing wave numbers, the latter contributions can be termed as "accelerated". It should be clear that such contributions notably improve the convergence of truncated series.

\section{c) Backward step}

The solution finally appears - for any $N$ - as the announced superposition of an ordinary response of the first $N$ modes, a pseudo-static term, $\eta_{N}$, and an "accelerated" response of the higher modes, from $N+1$ to $\infty$. In particular, for $N=1$ the proposed formulae give the solution as the superposition of the Helmholtz $0 \mathrm{~Hz}$ response, a pure static term, $\eta_{1}$, solution to

$$
\left\{\begin{array}{l}
\frac{\mathrm{d}^{2} \eta_{1}}{\mathrm{~d} x^{2}}=-\rho \gamma \psi_{1}(L) \psi_{1}(x), \frac{\mathrm{d} \eta_{1}}{\mathrm{~d} x}(0)=0, \frac{\mathrm{d} \eta_{1}}{\mathrm{~d} x}(L)=-\rho \gamma \\
\sqrt{\frac{1}{L}} \int_{0}^{L} \eta_{1} \mathrm{~d} x=0
\end{array}\right.
$$

and the "accelerated" modal response of all subsequent modes.

Since $\psi_{1}(x)=1 / \sqrt{L}$, the general solution to problem (22) is a polynomial of degree $2, \eta_{1}=-\frac{\rho \gamma}{2 L} x^{2}+b x+c$. The two boundary conditions bring $b=0$ but leave the 

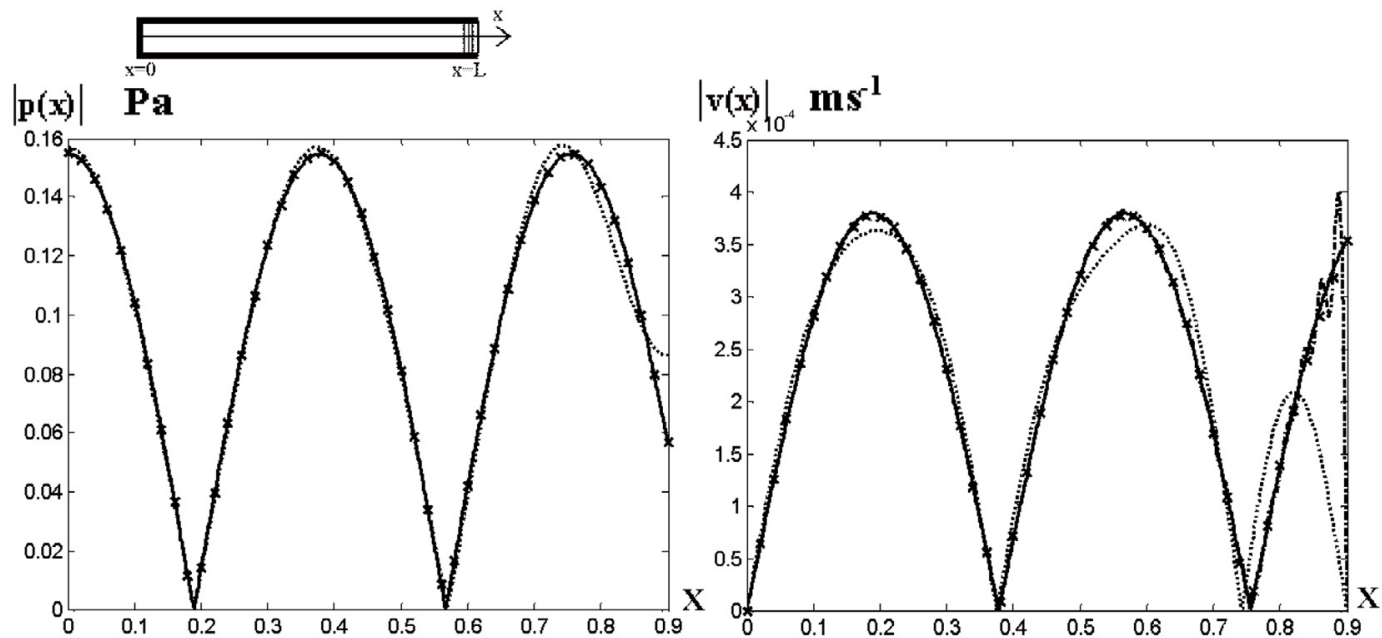

Fig. 2. Magnitudes at $450 \mathrm{~Hz}$ of acoustic pressure and velocity in a closed $90 \mathrm{~cm}$-tube: $\times$ exact; - accelerated 8-mode superposition; $\cdots$ ordinary 8 -mode sum; -.--ordinary 70-mode sum.

solution undetermined while the complementary integral condition brings

$$
\left[\frac{-\rho \gamma x^{3}}{6 L}+c x\right]_{0}^{L}=0 \Rightarrow c=\frac{\rho \gamma L}{6}
$$

The acoustic pressure, $p$, and velocity, $\nu$, can thus be written as

$$
\left\{\begin{array}{r}
p(x)=\frac{\rho \gamma}{k^{2} L}-\frac{\rho \gamma}{2 L}\left(x^{2}-\frac{L^{2}}{3}\right)+\sum_{m \geq 2} \frac{2(-1)^{m-1}(-\rho \gamma)}{L} \\
\quad \times \frac{k^{2}}{k_{m}^{2}\left(k_{m}^{2}-k^{2}\right)} \cos \frac{(m-1) \pi x}{L} \\
\nu(x)=\frac{-i \gamma}{\omega L} x+\sum_{m \geq 2} \frac{2(-1)^{m-1}(i \gamma)(m-1) \pi}{\omega L^{2}} \\
\times \frac{k^{2}}{k_{m}^{2}\left(k_{m}^{2}-k^{2}\right)} \sin \frac{(m-1) \pi x}{L}
\end{array}\right.
$$

\subsubsection{Numerical example}

Figure 2 shows the acoustic pressure, $p$, and velocity, $\nu$, that are developed at $450 \mathrm{~Hz}$ inside a closed tube of the length $L=0.90 \mathrm{~m}$, when it is filled up with air $\left(\rho=1.2 \mathrm{~kg} \cdot \mathrm{m}^{-3}, c=340 \mathrm{~m} \cdot \mathrm{s}^{-1}\right)$ and subjected to a unit acceleration $\gamma=1 \mathrm{~m} . \mathrm{s}^{-2}$ at $x=L$. Applying formulae (19) up to the 8th mode at $1322 \mathrm{~Hz}$, leads to results that are in very good agreement with the analytical solution,

$$
p(x)=\frac{\rho \gamma}{k \sin k L} \cos k x, \nu(x)=\frac{-i \gamma \sin k x}{\omega \sin k L}
$$

In contrast, the 8-mode ordinary summation leads to totally inaccurate results while the ordinary summation up to the 70th mode at $13033 \mathrm{~Hz}$ leads to Gibbs oscillations at the end of the tube.

\subsection{Open tube}

\subsubsection{General equations}

Because of its importance in practical or computational acoustics, the case of open tubes - satisfying $p=0$, instead of $\mathrm{d} p / \mathrm{d} x=0$, at $x=0$ - is now considered.

Equation and boundary conditions (10) are now replaced by

$$
\frac{\mathrm{d}^{2} p}{\mathrm{~d} x^{2}}+k^{2} p(x)=0, \quad p(0)=0, \quad \frac{\mathrm{d} p}{\mathrm{~d} x}(L)=-\rho \gamma
$$

Under the normalization conditions of Section 3.1.1, eigenmodes, and frequencies, $\phi_{m}, h_{m}$, satisfying

$$
\frac{\mathrm{d}^{2} \phi_{m}}{\mathrm{~d} x^{2}}+h_{m}^{2} \phi_{m}(x)=0, \quad \phi_{m}(0)=0, \quad \frac{\mathrm{d} \phi_{m}}{\mathrm{~d} x}(L)=0
$$

are given by

$$
\begin{aligned}
\phi_{m}(x) & =B_{m} \sin h_{m} x=B_{m} \sin \left(-\frac{\pi}{2}+m \pi\right) \frac{x}{L} \\
\text { with } \quad B_{m} & =\sqrt{\frac{2}{L}}
\end{aligned}
$$

\subsubsection{Analysis by the method of orthocomplement}

Using the second Green formula leads to $\left\langle p \mid \phi_{m}\right\rangle=$ $\frac{-\rho \gamma \phi_{m}(L)}{\left(h_{m}^{2}-k^{2}\right)}$, from which the modal expansion of $p$ can be written as

$$
p(x)=\sum_{m=1}^{N}-\rho \gamma \frac{\phi_{m}(x) \phi_{m}(L)}{\left(h_{m}^{2}-k^{2}\right)}+\varepsilon_{N}
$$




\section{a) Forward step}

Differentiating Equation (27) with respect to $x$ and using Equations (24), (25), show the orthocomplement, $\varepsilon_{N}$, to satisfy

$$
\begin{aligned}
\frac{\mathrm{d}^{2} \varepsilon_{N}(x)}{\mathrm{d} x^{2}}+k^{2} \varepsilon_{N}(x) & =-\sum_{m=1}^{N} \rho \gamma \phi_{m}(L) \phi_{m}(x) \\
\varepsilon_{N}(0) & =0, \frac{\mathrm{d} \varepsilon_{N}}{\mathrm{~d} x}(L)=-\rho \gamma
\end{aligned}
$$

Following the proposed methodology, problem (28) can be split up into two nested sub-problems, (A), (B), according to

$$
\varepsilon_{N}=\eta_{N}+\rho_{N}\left\{\begin{array}{c}
\frac{\mathrm{d}^{2} \eta_{N}}{\mathrm{~d} x^{2}}=\sum_{m=1}^{N}-\rho \gamma \phi_{m}(L) \phi_{m}(x), \eta_{N}(0)=0, \\
\frac{\mathrm{d} \eta_{N}}{\mathrm{~d} x}(L)=-\rho \gamma \quad(\mathrm{A}) \\
\frac{\mathrm{d}^{2} \rho_{N}}{\mathrm{~d} x^{2}}+k^{2} \rho_{N}=-k^{2} \eta_{N}, \rho_{N}(0)=0, \\
\frac{\mathrm{d} \rho_{N}}{\mathrm{~d} x}(L)=0 \quad(\mathrm{~B})
\end{array}\right.
$$

In contrast with the previous section, problem (A) is no longer of the Neumann type and does not present any singularity. Integrating twice and identifying constants indeed bring the only solution

$$
\begin{aligned}
\eta_{N}(x)= & -\rho \gamma x\left(1-\sum_{m=1}^{N} \phi_{m}(L) \int_{0}^{L} \phi_{m}(y) \mathrm{d} y\right) \\
& -\rho \gamma \sum_{m=1}^{N} \phi_{m}(L) \int_{0}^{x} \int_{0}^{y} \phi_{m}(z) \mathrm{d} z \mathrm{~d} y
\end{aligned}
$$

\section{b) Coordination step}

Problem (B) being a smooth dynamical problem with homogeneous boundary conditions, its solution, $\rho_{N}$, admits the modal expansion

$$
\rho_{N}(x)=\sum_{m=1}^{\infty}\left\langle\rho_{N} \mid \phi_{m}\right\rangle \phi_{m}(x)
$$

Substituting for the boundary values and derivatives of $\rho_{N}, \phi_{m}$, from conditions (29), (25) into the second Green formula for $\rho_{N}, \phi_{m}$, brings as usual

$$
\left\langle\rho_{N} \mid \phi_{m}\right\rangle=\frac{k^{2}}{h_{m}^{2}-k^{2}}\left\langle\eta_{N} \mid \phi_{m}\right\rangle
$$

while substituting for the boundary values and derivatives of $\eta_{N}, \phi_{m}$, from the same conditions (29), (25) into the second Green formula for $\eta_{N}, \phi_{m}$, brings

$$
\left\langle\eta_{N} \mid \phi_{m}\right\rangle=\left\{\begin{array}{l}
0 \quad \text { if } 1 \leq m \leq N \\
\frac{-\rho \gamma \phi_{m}(L)}{h_{m}^{2}} \quad \text { if } m>N
\end{array}\right.
$$

Coordination of the two sub-problems, (A), (B), is here greatly simplified by the absence of the $0 \mathrm{~Hz}$ singularity that introduced an ineffective identity $0=0$ for $m=1$. It is thus possible to substitute $\left\langle\eta_{N} \mid \phi_{m}\right\rangle$ from (33) into (32)

$$
\begin{aligned}
\left\langle\rho_{N} \mid \psi_{m}\right\rangle=\frac{k^{2}}{k_{m}^{2}-k^{2}} & \left\langle\eta_{N} \mid \phi_{m}\right\rangle \\
& =\left\{\begin{array}{l}
0 \text { if } 1 \leq m \leq N \\
-\frac{k^{2}}{h_{m}^{2}} \frac{\rho \gamma \phi_{m}(L)}{h_{m}^{2}-k^{2}} \text { if } m>N
\end{array}\right.
\end{aligned}
$$

\section{c) Backward step}

For each possible $N$, the solution still appears as the announced superposition of an ordinary modal sum based on the first $N$ modes, a pseudo-static term, $\eta_{N}$, and an "accelerated" response based on higher modes. By a convenient choice of $N$, every mode of order $m, m=1, \ldots N$, can either be placed inside an ordinary summation, if $m \leqslant N$, or in the accelerated part, if $N<m$. The minimal formula of order $N=0$ gives the solution as the superposition of a pure static term, $\eta_{0}(x)=-\rho \gamma x$, deduced from Equation (30), and a full accelerated modal series, the acoustic pressure and velocity being then written

$$
\left\{\begin{aligned}
p(x)= & -\rho \gamma x \\
& +\sum_{m \geq 1} \frac{2(-1)^{m-1}(-\rho \gamma)}{L} \frac{k^{2}}{h_{m}^{2}\left(h_{m}^{2}-k^{2}\right)} \sin \left(h_{m} x\right) \\
\nu(x)= & (-i / \omega) \\
& \times\left(\gamma+\sum_{m \geq 1} \frac{2 \gamma(-1)^{m-1}}{L} \frac{k^{2}}{\left.h_{m}^{(} h_{m}^{2}-k^{2}\right)} \cos \left(h_{m} x\right)\right)
\end{aligned}\right.
$$

\subsubsection{Numerical example}

Figure 3 shows the acoustic pressure, $p$, and velocity, $v$, at $450 \mathrm{~Hz}$ inside the preceding $90 \mathrm{~cm}$-tube, when the pressure at $x=0$ is forced to 0 and the acceleration at $x=L$ is $\lambda=1 \mathrm{~m} . \mathrm{s}^{-2}$. Formulae (35) up to the 8 th mode at $1417 \mathrm{~Hz}$, lead to results in very good agreement with the analytical solution,

$$
p(x)=\frac{-\rho \gamma}{k \cos k L} \sin k x, \nu(x)=\frac{-i \gamma}{\omega \cos k L} \cos k x
$$

Ordinary modal superpositions lead to better results than for the closed tube. The 8-mode ordinary summation remains however inaccurate and Gibbs oscillations can still be seen on the ordinary summation up to the 70th mode at $13128 \mathrm{~Hz}$.

\section{3D acoustics}

\subsection{Complements in linear algebra}

A complete extension of the 1D academic studies of Section 3 to $2 \mathrm{D}$ or $3 \mathrm{D}$ problems can be found in [8], leading to the family of hybrid modal expressions defined by 

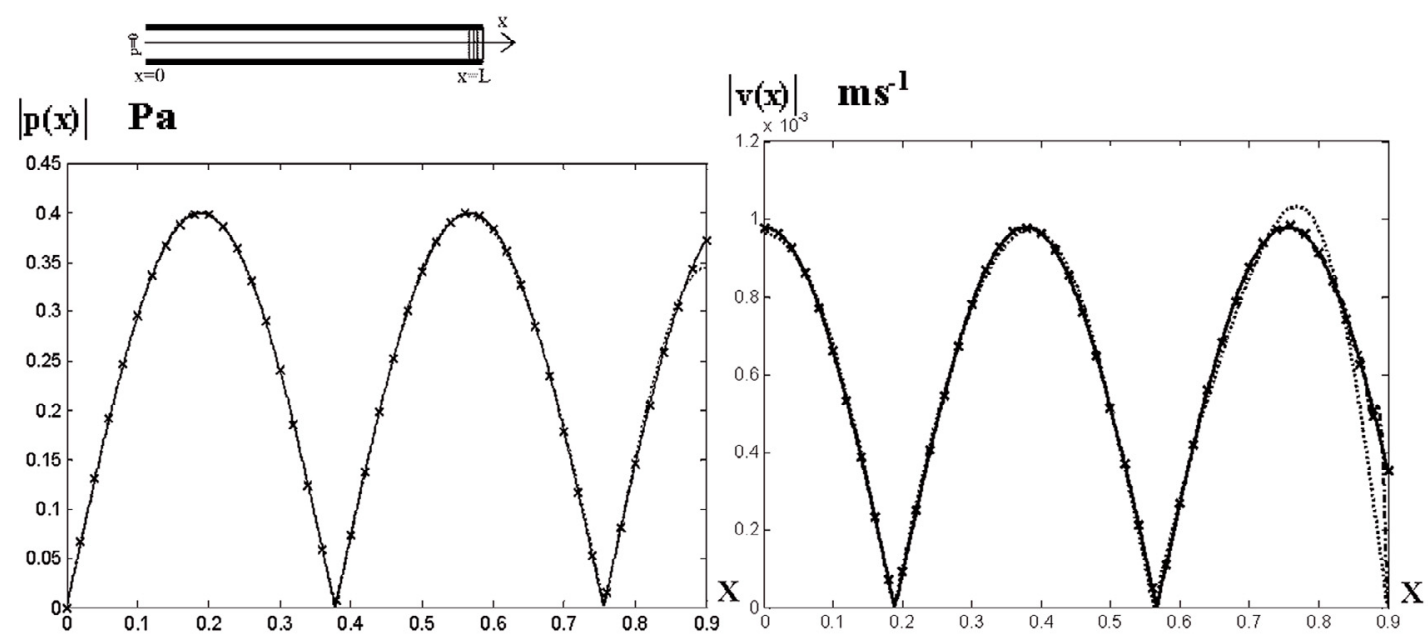

Fig. 3. Amplitudes at $450 \mathrm{~Hz}$ of acoustic pressure and velocity in a closed $90 \mathrm{~cm}$-tube: $\times$ exact; - accelerated 8-mode superposition; $\cdots$ ordinary 8-mode sum; -.--ordinary 70-mode sum.

formulae (1) and (3). Finite-element approximations lead to special difficulties in the numerical solution of singular pseudo-static problems. The present paragraph gives thus a brief account of some useful pseudo-inverse results taken from [8].

\subsubsection{Overdeterminated rectangular systems}

Any system in the form $\mathbf{S} \mathbf{x}=\mathbf{f}$, with $\mathbf{S}$ a rectangular $(p, q)$-matrix, $p>q$, satisfying the maximum rank condition, $\operatorname{rank}(\mathbf{S})=\min (p, q)=q$, admits a Moore-Penrose pseudo-inverse solution

$$
\mathbf{x}^{*}=\left(\mathbf{S}^{T} \mathbf{S}\right)^{-1} \mathbf{S}^{T} \mathbf{f}
$$

that is obtained by minimizing $F(\mathbf{x})=\frac{1}{2}\|\mathbf{S} \mathbf{x}-\mathbf{f}\|^{2}[10]$

As a consequence, the orthogonal projector on the subspace generated by the columns of $\mathbf{S}$ is

$$
\boldsymbol{\Pi}=\mathbf{S}\left(\mathbf{S}^{T} \mathbf{S}\right)^{-1} \mathbf{S}^{T}
$$

The minimization can be made with the metric associated with an arbitrary symmetric positive matrix, $\mathbf{B}$. This leads to general expressions of $\mathbf{B}$-pseudo-inverses as

$$
\mathbf{x}^{* \mathbf{B}}=\left(\mathbf{S}^{T} \mathbf{B} \mathbf{S}\right)^{-1} \mathbf{S}^{T} \mathbf{B} \mathbf{f}
$$

and of $\mathbf{B}$-projectors as

$$
\mathbf{\Pi}^{\mathbf{B}}=\mathbf{S}\left(\mathbf{S}^{T} \mathbf{B} \mathbf{S}\right)^{-1} \mathbf{S}^{T} \mathbf{B}
$$

\subsubsection{Singular square systems}

The singular linear systems that most commonly arise in mechanical applications, are in the form

$$
\mathbf{K} \mathbf{x}=\mathbf{f}
$$

with $\mathbf{K}=\mathbf{K}^{T}$, $\operatorname{det}(\mathbf{K})=0$. Matrix $\mathbf{K}$ is generally a stiffness or $\mathbf{H}$-matrix, and the kernel, $\operatorname{Ker}(\mathbf{K})$, can be constituted, by example, by rigid motions in floating mechanical structures or constants in Neumann acoustic problems. Let $\mathbf{S}$ denote a spanning matrix of $\operatorname{Ker}(\mathbf{K})$, i.e. a $p \times q$ matrix of maximum rank, such that

$$
\operatorname{Ker}(\mathbf{K})=\operatorname{Im}(\mathbf{S})=\left\{\mathbf{y} \in R^{p} \mid \exists \beta \in R^{q}: \mathbf{y}=\mathbf{S} \beta\right\}
$$

System (41) is soluble if $\mathbf{f} \in \operatorname{Im}(\mathbf{K})$, or - by a known theorem of Linear Algebra - if it is an element orthogonal to $\operatorname{Ker}(\mathbf{K})$, satisfying the integrability condition:

$$
\mathbf{S}^{T} \mathbf{f}=0
$$

In this case, the system admits an infinity of solutions of the form

$$
\mathbf{x}=\mathbf{x}_{0}+\mathbf{S} \alpha
$$

with $\mathbf{x}_{0}$ a distinguished solution and $\mathbf{S} \alpha, \alpha \in R^{q}$, an arbitrary element of $\operatorname{Ker}(\mathbf{K})$.

The point is to produce additional conditions or relations - say $\mathbf{A} \mathbf{x}=0$ - that could constrain the initial system to deliver a well-posed problem

$$
\left\{\begin{array}{l}
\mathbf{K} \mathbf{x}=\mathbf{f} \\
\mathbf{A x}=\mathbf{0}
\end{array}\right.
$$

whose unique solution could be taken as the special solution $\mathbf{x}_{0}$. Matrix $\mathbf{K}$ is a $p \times p$ matrix with rank $p-q$, since $q$, by (42), is the dimension of $\operatorname{Ker}(\mathbf{K})$. Additional restrictions should thus bring $q$ independent relations compatible with system (41). Given an arbitrary positive definite $p \times p$ matrix $\mathbf{B}$, the very simple choice $\mathbf{A}=\mathbf{S}^{T} \mathbf{B}$, that consists in imposing on the solution to be $\mathbf{B}$ - orthogonal to $\operatorname{Ker}(\mathbf{K})$, does not only bring the correct number of equations but also ensures compatibility and uniqueness. Indeed, the corresponding $\mathbf{x}_{0}-$ or say $\mathbf{x}_{M}$ - is the only point of intersection of the set of solutions (44) with a 
complementary B-orthogonal subspace. The corresponding systems

$$
\left\{\begin{array}{l}
\mathbf{K} \mathbf{x}=\mathbf{f} \\
\mathbf{S}^{T} \mathbf{B} \mathbf{x}=\mathbf{0}
\end{array}\right.
$$

reveals themselves to be quite common in mechanical applications: it can be verified, by example, that $\mathbf{B}=\mathbf{1}$ corresponds to the SVD solution; while $\mathbf{B}=\mathbf{M}$, with $\mathbf{M}$ the mass matrix of a floating vibrating structure, corresponds to the "Inertia Relief" solution.

Robust projector methods have been proposed in [8] to solve such systems of linearly dependent equations. The SVD solution is given by

$$
\left.\begin{array}{l}
\mathbf{K} \mathbf{x}=\mathbf{f} \\
\mathbf{S}^{T} \mathbf{x}=\mathbf{0}
\end{array}\right\} \Leftrightarrow \mathbf{x}^{*}=(\lambda \boldsymbol{\Pi}+\mathbf{K})^{-1} \mathbf{f}
$$

where $\lambda$ is an arbitrary non-zero number and $\boldsymbol{\Pi}=$ $\mathbf{S}\left(\mathbf{S}^{T} \mathbf{S}\right)^{-1} \mathbf{S}^{T}$, the orthogonal projector $(38)$ on $\operatorname{Ker}(\mathbf{K})$, - with the somehow amazing property that $\mathbf{x}^{*}$ does not depend on $\lambda$ provided the integrability condition $\mathbf{S}^{T} \mathbf{f}=0$ be fulfilled. General solutions for $\mathbf{B} \neq \mathbf{1},-$ like the "Inertia Relief" one, - can be deduced from the SVD solution according to

$$
\left.\begin{array}{l}
\mathbf{K} \mathbf{x}=\mathbf{f} \\
\mathbf{S}^{T} \mathbf{B} \mathbf{x}=\mathbf{0}
\end{array}\right\} \Leftrightarrow \mathbf{x}=\left(\mathbf{1}-\mathbf{\Pi}^{\mathbf{B}}\right)(\lambda \mathbf{\Pi}+\mathbf{K})^{-1} \mathbf{f}
$$

where $\mathbf{\Pi}^{\mathbf{B}}=\mathbf{S}\left(\mathbf{S}^{T} \mathbf{B S}\right)^{-1} \mathbf{S}^{T} \mathbf{B}$ denotes the $\mathbf{B}$-orthogonal projector (40) on $\operatorname{Ker}(\mathbf{K})$.

\subsection{Finite-element formulation}

For the purpose of numerical analysis, matrices $\mathbf{H}$ and Q, must be classically synthesized, in such a way that

$$
\int_{\Omega} \nabla f \nabla g \mathrm{~d} x=\mathbf{f} \mathbf{H} \mathbf{g}, \int_{\Omega} f g \mathrm{~d} x=, \mathbf{f} \mathbf{Q} \mathbf{g}
$$

for every pair $f, g$ of functions in $H^{1}(\Omega)$, approximated by nodal vectors $\mathbf{f}$ and $\mathbf{g}$. Numerical modes and eigenfrequencies can then be defined by solving

$$
\left(\mathbf{H}-\omega_{k}^{2} \mathbf{Q}\right) \mathbf{\Psi}_{k}=0
$$

Assembling the pseudo-static problem (3)

$$
\begin{gathered}
\nabla^{2} \eta_{N}=\sum_{m=1}^{N} \psi_{m}\left(\int_{\partial \Omega} \psi_{m} D \mathrm{~d} s\right) \text { in } \Omega, \frac{\partial \eta_{N}}{\partial n}=D \\
\frac{\partial \eta_{N}}{\partial n}=D
\end{gathered}
$$

is a little less trivial.

The first step consists in building the associated weak formulation of Equation (51). Namely, the field equation, multiplied by $\delta \eta \in H^{1}(\Omega)$, must be integrated over the domain and transformed by means of Green's formula to take the boundary condition into account. The solution can thereby be characterized by the variational equality

$$
\forall \delta \eta: \int_{\Omega} \nabla(\delta \eta) . \nabla \eta_{N} \mathrm{~d} \nu+\int_{\Omega} \delta \eta g_{N} \mathrm{~d} \nu-\int_{\partial \Omega} \delta \eta D \mathrm{~d} s=0
$$

where $D$ is the prescribed boundary data and $g_{N}$ is defined, as in $1 \mathrm{D}$ case by

$$
g_{N}=\sum_{m=1}^{N} \psi_{m}\left(\int_{\partial \Omega} \psi_{m} D \mathrm{~d} s\right)
$$

Source vectors $\mathbf{F}$ and $\mathbf{G}_{N}$, respectively on $\partial \Omega$ and inside $\Omega$, being defined such that

$$
\delta \eta^{T} \mathbf{F} \simeq \int_{\partial \Omega} \delta \eta D \mathrm{~d} s, \delta \eta^{T} \mathbf{G}_{N} \simeq \int_{\Omega} \delta \eta g_{N} \mathrm{~d} \nu
$$

the finite element discretization of Equation (54) gives the nodal pseudo-static pressure vector $\eta_{N}$ as a solution to

$$
\forall \delta \eta: \delta \eta^{T} \mathbf{H} \eta_{N}=\delta \eta^{T} \mathbf{F}-\delta \eta^{T} \mathbf{G}_{N}
$$

or, in an equivalent matrix form, to

$$
\mathbf{H} \eta_{N}=\mathbf{F}-\mathbf{G}_{N}
$$

It will be supposed that the approximation is "conformal", i.e. that, up to minor numerical errors, $\mathbf{H}$ is a singular matrix with a 1D kernel and that the second member of Equation (57), - which comes from self-equilibrated source terms, $D$ and $g_{N}$ lies in $\operatorname{Im}(\mathbf{H})$.

The subsidiary condition $\int_{\Omega} \eta_{N} \mathrm{~d} \nu=0$ can be written

$$
\mathbf{S}^{T} \mathbf{Q} \eta_{N}=0
$$

with a vector of 1 's, $\mathbf{S}$, standing for a basis of the subspace of all constant functions. As a consequence, applying the pseudo-inversion formula (48) to the system (57), (58), results are deduced

$$
\eta_{N}=\left(1-\Pi_{Q}\right)(\lambda \Pi+\mathbf{H})^{-1}\left(\mathbf{F}-\mathbf{G}_{N}\right) \forall \lambda \neq 0
$$

It should be noted that $\Pi=\mathbf{S}\left(\mathbf{S}^{t} \mathbf{S}\right)^{-1} \mathbf{S}^{t}$ is a full constant matrix such that $\Pi_{m n}=1 / \bar{N}$ where $\bar{N}$ is the total number of nodes. The operator is ill-conditioned for small values of $\lambda$ since $\lambda \Pi+\mathbf{H} \simeq \mathbf{H}$, as well as for large values, since, in that case, $\lambda \Pi+\mathbf{H} \simeq \lambda \Pi$. The use of intermediate values of $\lambda$, of the order of magnitude $\bar{N}\|H\|$, is therefore highly recommended.

\subsection{Numerical example: simplified car compartment}

Figure 4 presents the complete 3D finite element modal analysis of a car compartment driven by a boundary source (diagram a). Despite of the fact that the 10th 


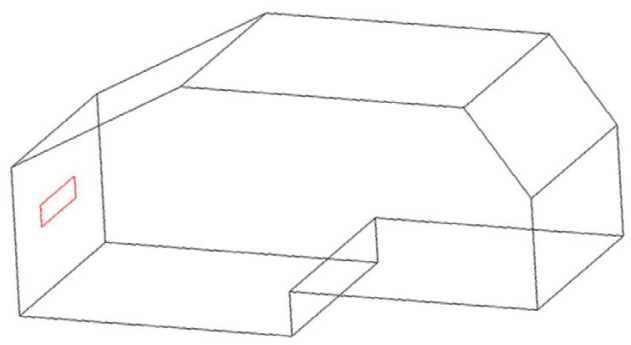

a
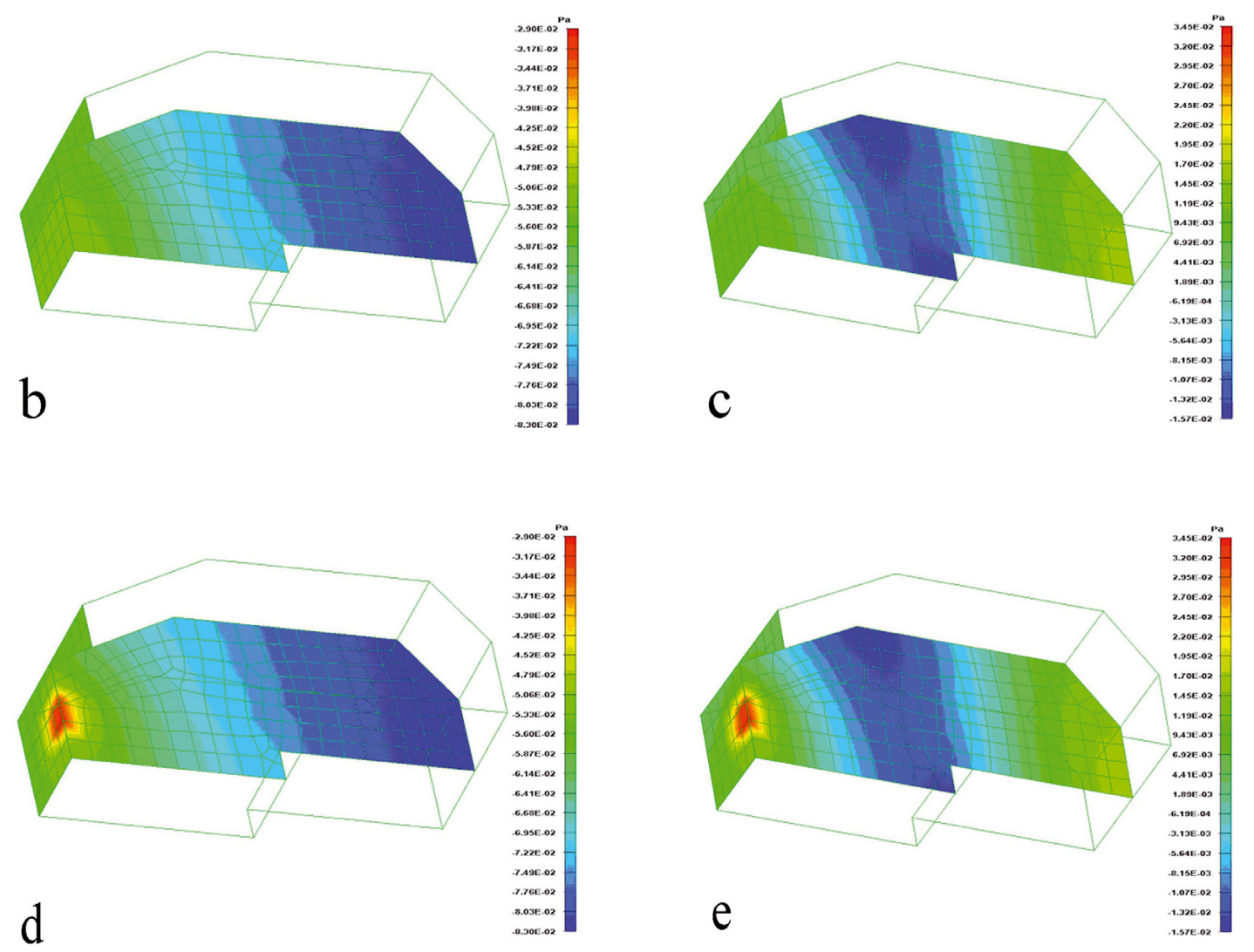

Fig. 4. Amplitudes of acoustic pressure inside a car compartment: a location of the source; $\mathbf{b}$, c ordinary $10-$ mode sum at $50 \mathrm{~Hz}$ and $300 \mathrm{~Hz}$; d, e accelerated 10-mode sum and direct responses at 50 and $300 \mathrm{~Hz}$.

eigen frequency is $623 \mathrm{~Hz}$, ordinary 10-mode summations at $50 \mathrm{~Hz}$ (diagram b) and $300 \mathrm{~Hz}$ (diagram c) give quite inaccurate results, while 10 -mode accelerated sums with correct static terms are in perfect agreement with direct responses (diagrams d, e).

\section{Conclusion}

Practical Green functions, that can be easily derived from the preceding acoustic fields, are of a great interest in the effective solution of a multitude of practical problems.

To fix ideas, when a resonator is placed at some point $\mathrm{A}$ on the boundary of a rigid cavity, the acoustic field at any point $\mathrm{B}$ in the modified cavity, $p_{B}^{(z)}$, and its value, $p_{B}^{(\infty)}$, in the initial rigid cavity only differ by the solution of a Neumann boundary problem with zero normal boundary velocity outside $\mathrm{A}$ and a temporarily unknown velocity, $\nu_{A}^{(z)}$, on A. The acoustic boundary impedance $Z_{B \leftarrow A}$ being defined as the pressure at $\mathrm{B}$ for a unit boundary velocity at $\mathrm{A}$, one thus finds: $p_{B}^{(z)}=p_{B}^{(\infty)}+Z_{B \leftarrow A} \nu_{A}^{(z)}$. Writing the conditions of continuity at A, classically leads to parametrized coupling formulae of the type

$$
p_{B}^{(z)}=p_{B}^{(\infty)}-Z_{B \leftarrow A}\left[Z_{A \leftarrow A}+z\right]^{-1} p_{A}^{(\infty)}
$$

that are presently investigated by an increasing number of authors $[11,15]$. 
Robust and efficient modal formulae have been presented that are now to be used in practical applications for the design and synthesis of complex resonators and the coupling of such structures with car or engine compartments.

\section{References}

[1] D. Gottlieb, S. Gottlieb, Spectral methods for discontinuous problems, Biennial Numerical Analysis Conference, NA03 2003, pp. 65-71 http://www.maths.dundee.ac. uk/ naconf/proc03/gottlieb.pdf

[2] Y.Y. Kim, J.H. Kang, Inability of free-interface modes in representing stress resultants near the free interface, J. Ib. Acoustics 122 (2000) 409-411

[3] Z.-Q. Qu, Hybrid expansion method for frequency responses and their sensitivities, Part I: undamped systems, J. Sound Vib. 231 (2000) 175-193

[4] M. Tournour, N. Atalla, Pseudostatic corrections for the forced vibroacoustic response of a structure-cavity system, J. Acoustical Soc. Amer. 107 (2000) 2379-2386

[5] M. Tournour, N. Atalla, O. Chiello, F. Sgard, Validation, performance, convergence and application of free interface component mode synthesis, Computer \& Structures 79 (2001) 1861-1876

[6] E. Balmès, Optimal Ritz vectors for component mode synthesis using the singular value decomposition, AIAA J. 34 (1996) 1256-1260
[7] O. Sauvage, Modélisation du comportement vibratoire d'un groupe moto-propulseur de 0 à $10000 \mathrm{~Hz}$, Thèse de doctorat de l'École Centrale de Paris, 2002 (in french)

[8] J.-M. Lagache, S. Assaf, C. Schulte, Finite element synthesis of structural or acoustic receptances in view of practical applications, J. Sound Vib. 310 (2008) 313-351

[9] J.N. Reddy, Applied functional analysis and variational methods in engineering, McGraw-Hill, New York, 1986, pp. 229-231

[10] G. Strang, Introduction to Applied Mathematics, Welleslay, Cambridge Press, 1986

[11] O. Tanneau, O. Sauvage, J.M. Lagache, Analyse vibratoire de modifications de structures. L'approche des cercles de Vincent revisitée, $\mathrm{XV}^{\mathrm{e}}$ Colloque Vibrations, Chocs et Bruits, École Centrale de Lyon, 14-16 juin 2006 (in french)

[12] M.G. Tehrani, W. Wang, C. Mares, J.E. Mottershead, The generalized Vincent circle in vibration suppression, J. Sound Vib. 292 (2006) 661-675

[13] M.G. Tehrani, W. Wang, C. Mares, J.E. Mottershead, Vibration Suppression Using Vincent's Circle, Proceeding of ISMA, 2004, pp. 603-661

[14] J.E. Mottershead, Structural modification for the assignment of zeros using measured receptances, Transactions of the American Society of Mechanical Engineers, J. Appl. Mech. 68 (2001) 791-798

[15] A.H. Vincent, A note on the properties of the variation of structural response with respect to a single structural parameter when plotted in the complex plane, Westland Helicopters Ltd., Report GEN/DYN/RES/010R, September 1973 